\title{
Design ANd Simulation of a FleXible CutTing MACHine for Roll Forming Product
}

\author{
Hong Seok Park, Duc Viet Dang, Gyu Bong Lee \& Ivica Veža
}
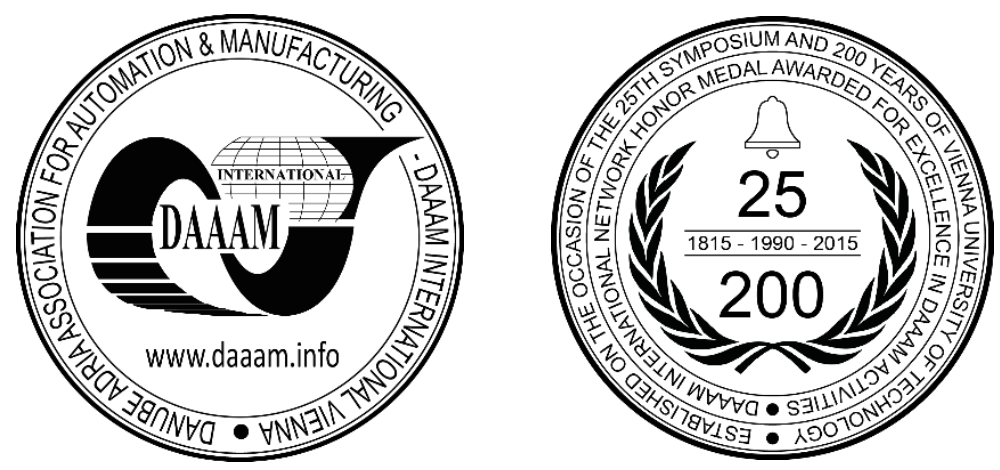

This Publication has to be referred as: Park, H[ong] S[eok]; Dang, D[uc] V[iet]; Lee, G[yu] B[ong] \& Veza, I[vica] (2016). Design and Simulation of a Flexible Cutting Machine for Roll Forming Product, Proceedings of the 27th DAAAM International Symposium, pp.0633-0644, B. Katalinic (Ed.), Published by DAAAM International, ISBN 978-3-90273408-2, ISSN 1726-9679, Vienna, Austria

DOI: $10.2507 / 27$ th.daaam.proceedings.092

\begin{abstract}
Development of a virtual simulation for machine is the imperative task to resolve time issue, improve cost saving and iterative improvements of physical prototype. The objective of the present paper was to design a flexible cutting machine and analyze the interactions between the machine structure and control system via a multi-body simulation (MBS) model and the virtual controller. Firstly, a reliable model of the flexible cutting machine was developed with the help of SOLIDWORKS and Automatic Dynamic Analysis of Mechanical System (ADAMS). Subsequently, a virtual controller used adaptive siding mode PID (ASMP) based on MATLAB/Simulink was designed. Finally, the co-simulation was performed to derive the machine movements under the effects of manufacturing conditions. The simulation results showed that the virtual mechanical model could precisely described the dynamic behavior of the flexible cutting machine. Furthermore, the proposed ASMP controller is robust regardless of the system parameter and disturbance variations. Therefore, this work is expected as a contribution to improve the prototype's performance of the flexible cutting machine.
\end{abstract}

Keywords: Flexible cutting machine; Roll forming; Multi-body simulation; Adaptive sliding mode; PID controller; Modelling and controlling.

\section{Introduction}

Roll forming parts are frequently applied to many kinds of industry and automobile sector. Along with straight roll forming product, some of roll forming products require a cured path (usually in arc form). In this case, the cut-off process is more difficult than that of straight products. The roll forming process requires the cutting operation to separate a long formed strip to a desired length. The cutting devices for straight roll forming parts are developed at a high level as illustrated in Fig. 1. However, the cutting device for curved products is still underdeveloped. Consequently, design a flexible and multi-functional cutting devices that can cut different cross section and product with arc or curvature in the longitudinal direction of roll forming product is a significant research. In order to develop a new product, the physical prototype can no longer suitable due to the time -consuming and cost-intensive manufacturing and testing to detect weak spots and optimize design [1]. Fortunately, literature reviews have performed that approaches based upon virtual prototype simulation, using well-defined material properties, 


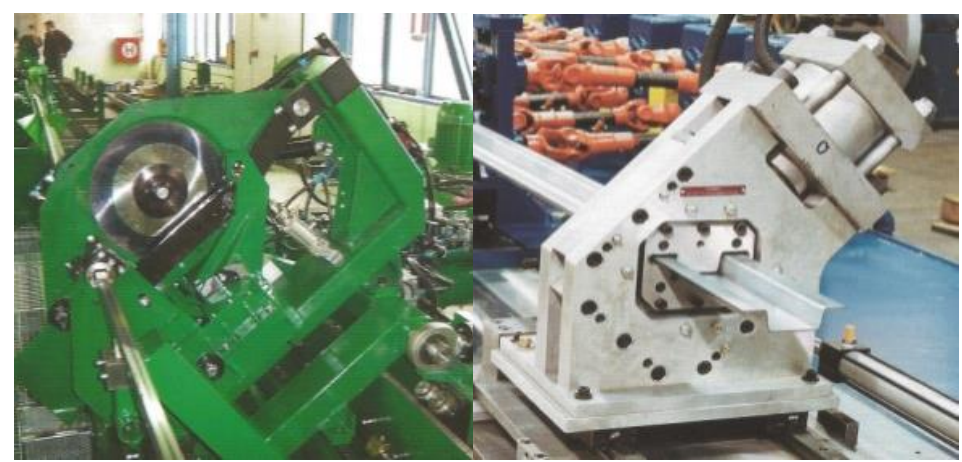

Fig. 1 The cutting devices for straight product

numerical models, and controllers, can be considered as an intelligent solution to obtain reliable results. With the support of the virtual prototype, the designer can analysis and optimize the mechanical design or the control design to achieve a perfect system, therefore it saves time and cost and reduces the risk of device damage caused by the conflict between both systems. There has been a considerable amount of research in the virtual prototype simulation which demonstrated the advantages of this method, such as Catalin et al. [2] simulated the windshield wiper system with the support of the virtual prototyping platform. Zouhaier [3] has built the crank slider mechanism to illustrate the behavior of the whole mechatronic system under different control strategies. Hajicek [4] presented a methodology for establishing virtual CNC machine tool. Moreover, the virtual prototypes of industrial equipment have been proposed to investigate dynamic behaviour in the initial stage [5] [6]. The results of the aforementioned efforts indicated that virtual prototyping techniques could be considered as a powerful approach to simulation and optimization the physical prototype of the new product. Therefore, this works attempted to develop a flexible cutting machine with the virtual prototype simulation.

This paper is organized as follows: the research methodology is described briefly in section 2. The concept of the flexible cutting machine is performed in section 3 and the multi-body system model is shown in section 4 . Section 5 will be explained the virtual controller. The results of co-simulation are showed section 6 and the conclusions are presented in this section 7.

\section{Research methodology}

The virtual prototype simulation platform includes CAD-Computer aided design, MBS-Multibody systems, FEAFinite element analysis and DFC-Design for control programs [2], as depicted in Fig. 2. The CAD environment is used to create the geometric model of the system from the concept of the manufacturer, which contains information about the mass and inertia properties of the rigid parts. The MBS software, which represents the central component of the virtual prototyping platform, is used for analyzing, optimizing and simulating the kinematic and dynamic behavior of the mechanical system. The FEA software is used for modeling flexible components. DFC is a software product which is used to design the control system. This software exchange information with the MBS software. The exchange process creates a closed loop in which the outputs of the MBS model are the inputs for the control system and conversely. The combination between the MBS and the DFC system will be implemented the virtual prototype.

The virtual prototype simulation of the flexible cutting machine is established by the combination ADAMS software and MATLAB/Simulink software as described in Fig. 3. With the concept of the machine, the modeling will be established in SOLIDWORKS and then exported to ADAMS software to create the multi-body system, with the possibility of virtual measurement of any parameters of any components in the virtual model. The controller will be designed in MATLAB environments which is well-known for designing a control system. The co-simulation model of both softwares will generate the virtual prototype which is performed the real behavior of the machine.

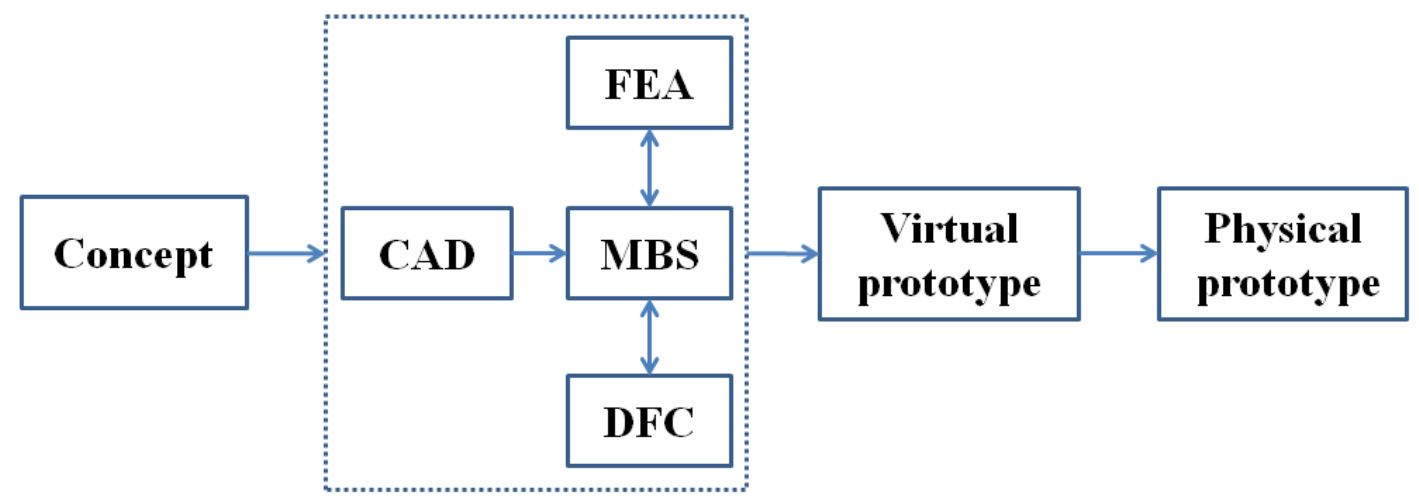

Fig.2. The virtual prototyping platform 


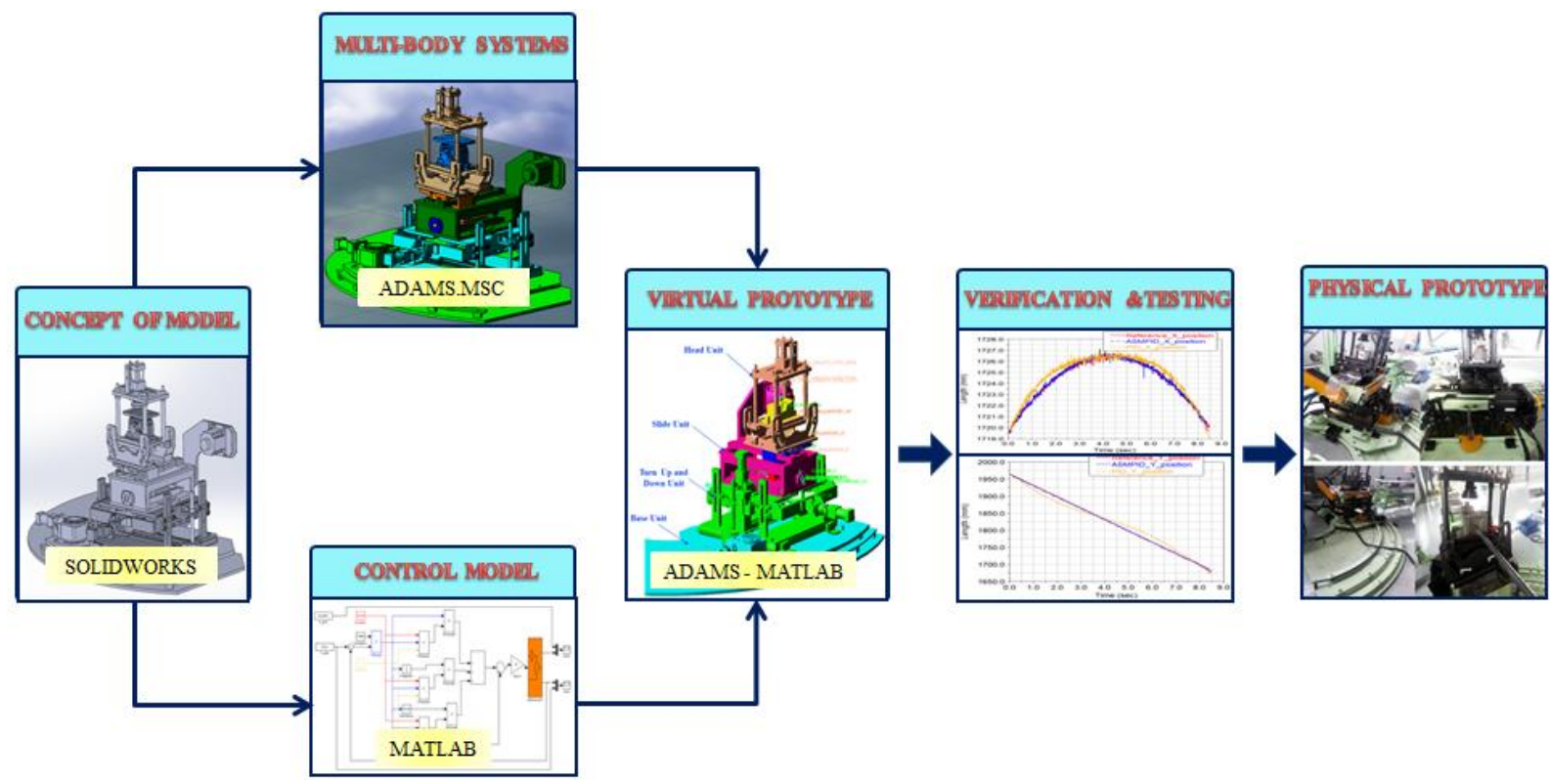

Fig. 3. The strategy for design and simulation of the flexible cutting machine.

\section{The concept of the flexible cutting machine}

Based on the straight forming machine, we attempt to develop the flexible cutting machine. This machine is used to manufacture the products require a cured path (usually in arc form), as shown in Fig. 4.
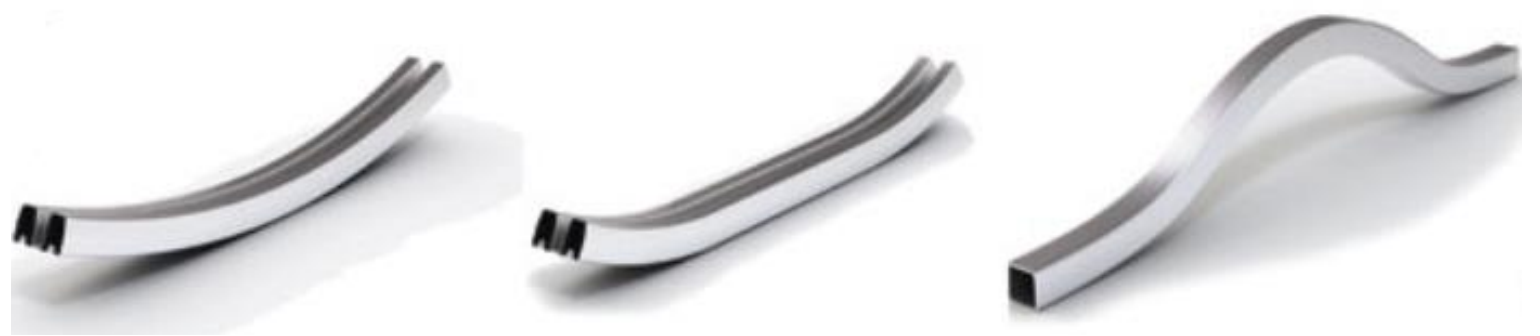

Fig. 4. The main types of work piece will be manufactured by the developed flexible cutting machine

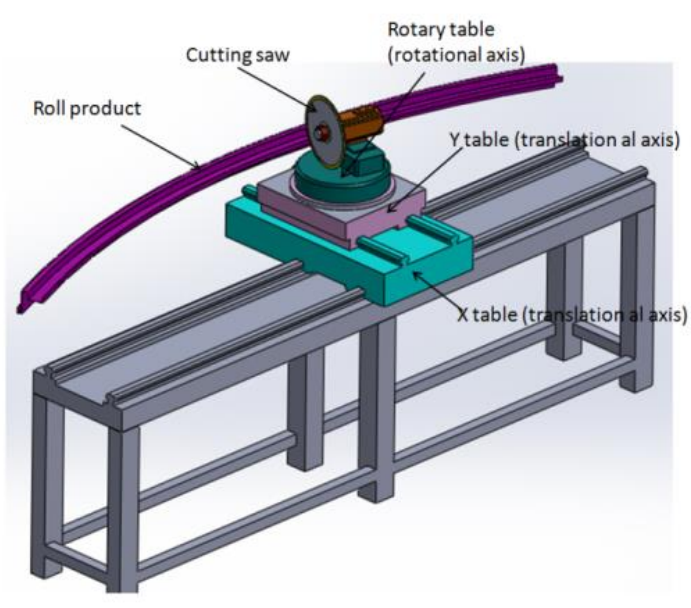

Fig. 5. The cutting machine with three degrees of freedom

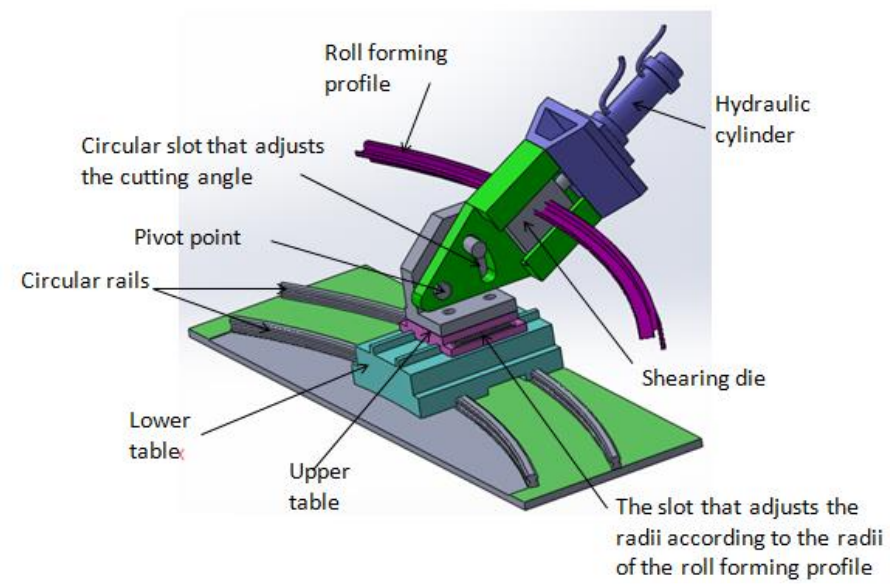

Fig. 6. The cutting machine with two degrees of freedom 


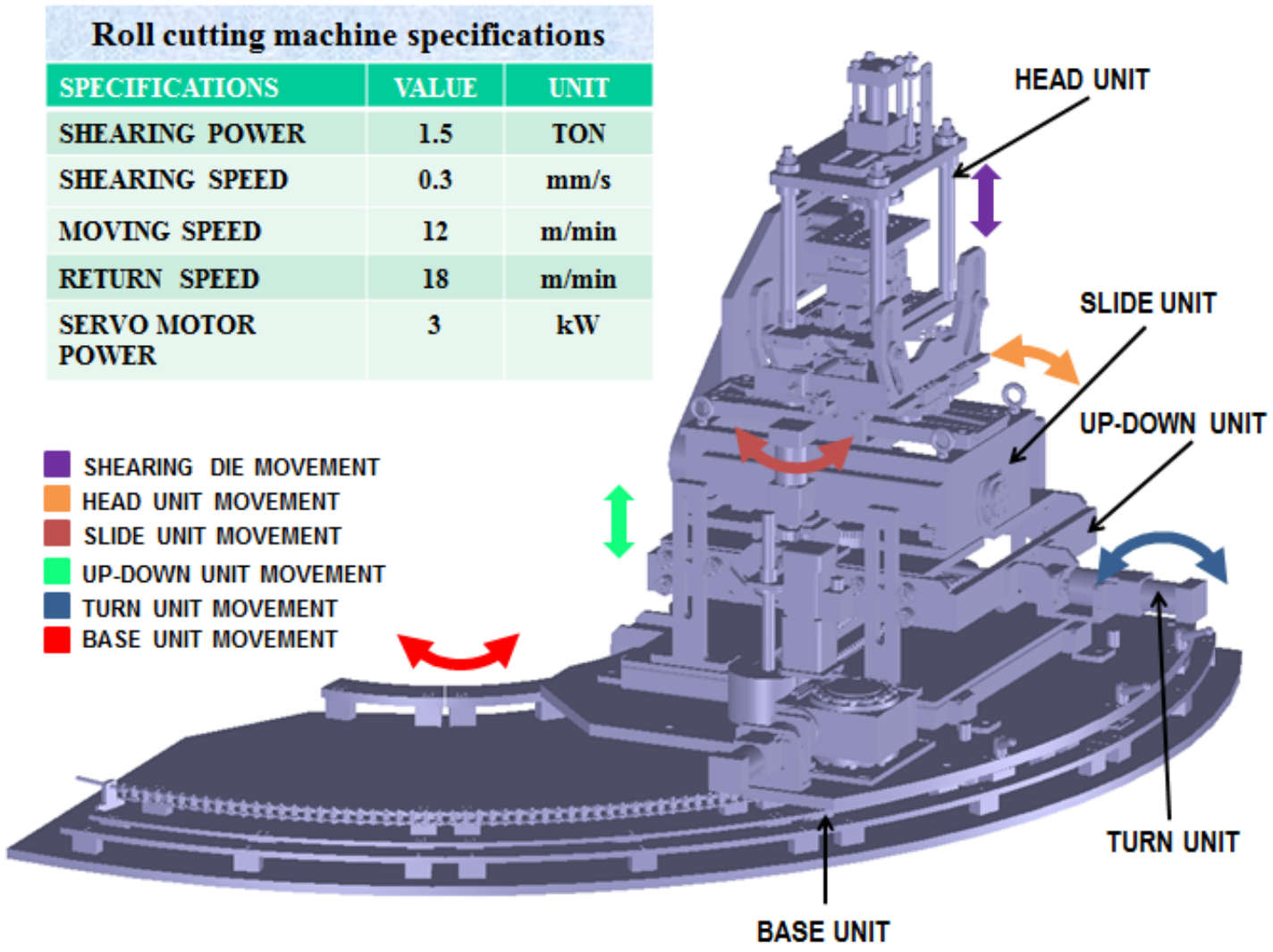

Fig. 7. The specifications and functionality of the flexible cutting machine

To satisfy the design requirements of the flexible cutting machine, two conceptual designs were proposed. The cutting machine using three degrees of freedoms and cutting saw was shown in Fig. 5, which includes two translations (longitudinal and traverse axis) and a rotational axis. The cutting movements are conducted based a combination of the machine bed and rotary table.

The conceptual machine using two degrees of freedoms is presented in Fig. 6. During forming process, the machine moves on two concentric circular rails. We can change the radius of the moving path by adjusting the position of the upper table. The cutting angle can be adjusted by means of a rotational pivot.

Based on the combination of the two proposed concepts, a concept design of the flexible cutting machine was proposed using five axes. The specifications and the construction of the roll cutting machine are shown in Fig. 7. During forming process, the base, turn, up-down, slide, and head unit are fixed. The head unit moves in the specific curved paths that is similar to the product profiles. The cutting movement at the end point of the contour is conducted by shearing die with the support of hydraulic cylinder.

The developed forming machine is a kind of moving cutting mechanism one. The cutting mechanism that not only generates the curved parts, but also cut off into accurate distance. The advantages of the flexible cutting machine are as follows compared to the conventional one:

- The machine can cut any kind of roll forming product regardless of straight or curve profile.

- Cutting distance is accurate because the mechanism is driven by servo motor.

- Hydraulic shearing mechanism with low noise.

\section{Multi-body system for the flexible cutting machine}

\subsection{Analytical modeling of a constrained multi-body mechanical system}

In this work, the flexible cutting machine was investigated as a multi-body mechanical system including of several rigid bodies. In the multi-body system [7], the generalized coordinates associated with rigid body $i$ is denoted in what follows by 


$$
\boldsymbol{q}_{i}=\left[\begin{array}{l}
p_{i} \\
\varepsilon_{i}
\end{array}\right]
$$

with $p_{i, \varepsilon_{i}}$ is the position and the orientation of a rigid body, respectively. For the system model containing $n b$ bodies, the vector $\boldsymbol{q}$ is represented to describe the position and orientation of each body at a given time in the system.

$$
\boldsymbol{q}=\left[\begin{array}{llll}
\boldsymbol{q}_{1}^{T} & \boldsymbol{q}_{2}^{T} & \ldots & \boldsymbol{q}_{n b}^{T}
\end{array}\right]^{T}
$$

Joints are regarded as constraints in the multi-body system which generally are obtained as

$$
\Phi=\Phi(\boldsymbol{q}, t)=0
$$

The entire constraint equations imposed by the joints are showed as

$$
\Phi(\boldsymbol{q}, t)=\left[\begin{array}{llll}
\Phi_{1}^{T}(\boldsymbol{q}, t) & \Phi_{2}^{T}(\boldsymbol{q}, t) & \ldots & \Phi_{n j}^{T}(\boldsymbol{q}, t)
\end{array}\right]^{T}
$$

where, $n j$ is the number of joints in the system. By taking one time derivative of the position kinematic constraint equation (4), the velocity kinematic constraint equations are expressed as follows

$$
\Phi_{q}(\boldsymbol{q}, t) \dot{\boldsymbol{q}}=-\Phi_{t}(\boldsymbol{q}, t)
$$

By taking one time derivative of the kinematic constraint equations Eqn (5), the acceleration kinematic constraint equations are obtained as

$$
\Phi_{q}(\boldsymbol{q}, t) \ddot{\boldsymbol{q}}=-\left(\Phi_{q} \dot{\boldsymbol{q}}\right)_{q} \dot{\boldsymbol{q}}-2 \Phi_{q t} \dot{\boldsymbol{q}}-\Phi_{t t}(\boldsymbol{q}, t)=\gamma
$$

The Lagrange multipliers form of the constraints acting on the multi body system can be written as

$$
\boldsymbol{M} \ddot{\boldsymbol{q}}+\Phi_{q}^{T} \lambda=\boldsymbol{F}
$$

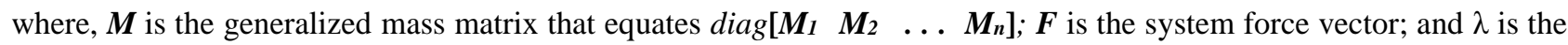
array of Lagrange multipliers. Combining Eqns (6) and (7), the complete set of constrained equations of motion can be written as follows

$$
\left[\begin{array}{cc}
\boldsymbol{M} & \Phi_{q}^{T} \\
\Phi_{q} & 0
\end{array}\right]\left[\begin{array}{l}
\ddot{\boldsymbol{q}} \\
\lambda
\end{array}\right]=\left[\begin{array}{l}
F \\
\gamma
\end{array}\right]
$$

Theories of multi-body systems are embedded to modelling and analysis using computational method. It allows the designer to observe how the mechanism functions without having to build an expensive real world model and doing labour intensive measurements.

\subsection{Multi-body system model}

In order to generate the multi-body system of the flexible cutting machine, all components or elements which have the shape and dimensions of the physical model are modeled as 3D solid by SOLIDWORKS. The geometry was transferred to ADAMS environment using the standard file parasolid. The modeling process is constructed as the following sequence for an easy modification in the design phase. Firstly, the geometrical parameters of the parts such as material properties must be defined, then mass and inertial matrices are generated automatically with main parameters in Table 1 . The flexible cutting machine model in ADAMS takes several aspects into account, such as gravity, contact constraints, joints, friction, motion and reference markers. All these definitions and setting must be defined properly for good approximation of real machine behavior in the virtual environment. The multi-bosy system of the flexible cutting machine in ADAMS software is depicted as Fig. 8 . 


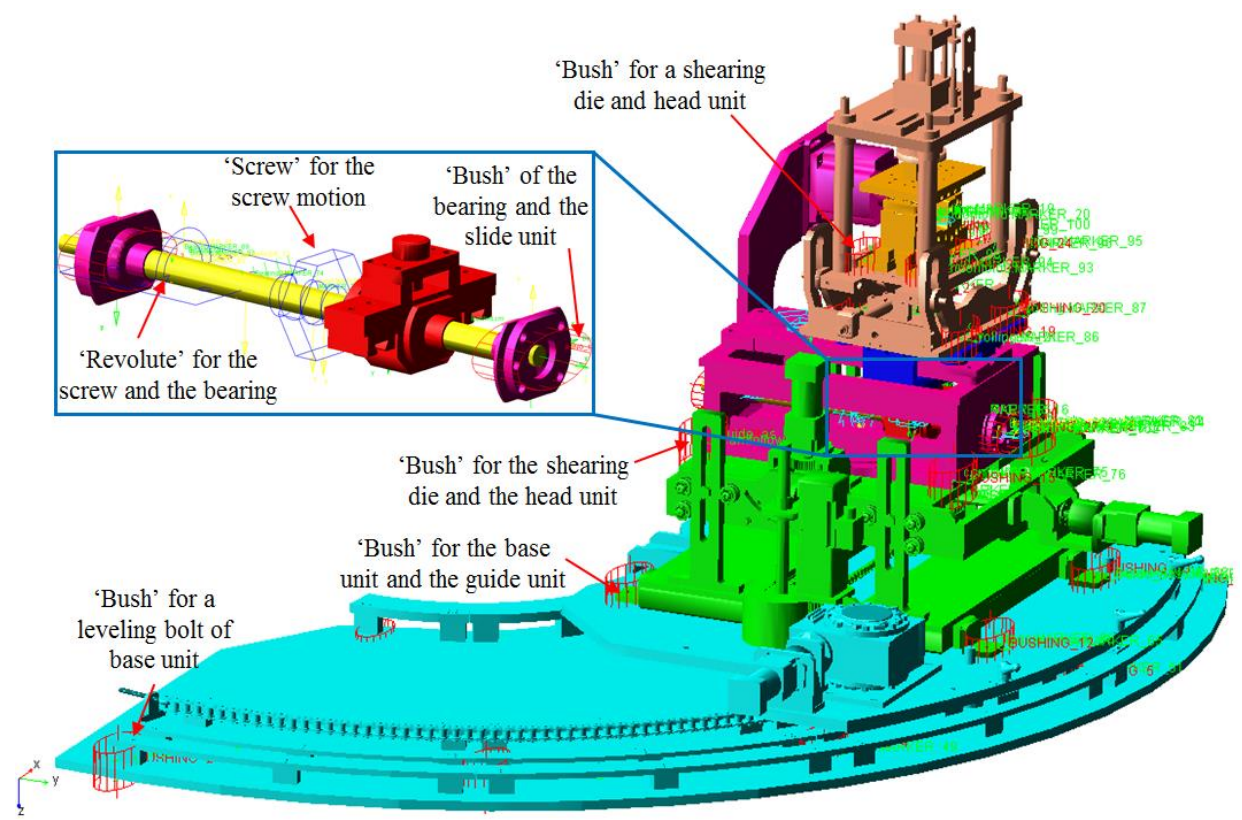

Fig. 8 . The multi-body system model of the flexible cutting machine in ADAMS. MSC

\begin{tabular}{|c|c|c|c|c|c|}
\hline Body name & Material & Mass $(\mathbf{k g})$ & $\mathbf{I}_{\mathbf{x x}}\left(\mathbf{k g .} \mathbf{m}^{\mathbf{2}}\right)$ & $\mathbf{I}_{\mathbf{y y}}\left(\mathbf{k g . \mathbf { m } ^ { 2 }}\right)$ & $\mathbf{I}_{\mathbf{z z}}\left(\mathbf{k g} . \mathbf{m}^{\mathbf{2}}\right)$ \\
\hline Base Unit & Cast steel & 1424.6 & 1063.65 & 796.51 & 278.63 \\
\hline Turn Up and Down Unit & Cast steel & 421.34 & 78.44 & 52.62 & 43.95 \\
\hline Slide Unit & Cast steel & 280.76 & 42.24 & 35.73 & 13.38 \\
\hline Head Unit & Cast steel & 172.69 & 857.78 & 699.35 & 1175.74 \\
\hline Shearing Die & Die steel & 38.01 & 188.19 & 154.14 & 258.99 \\
\hline
\end{tabular}

Table 1. Characteristics of main components of the flexible cutting machine in ADAMS
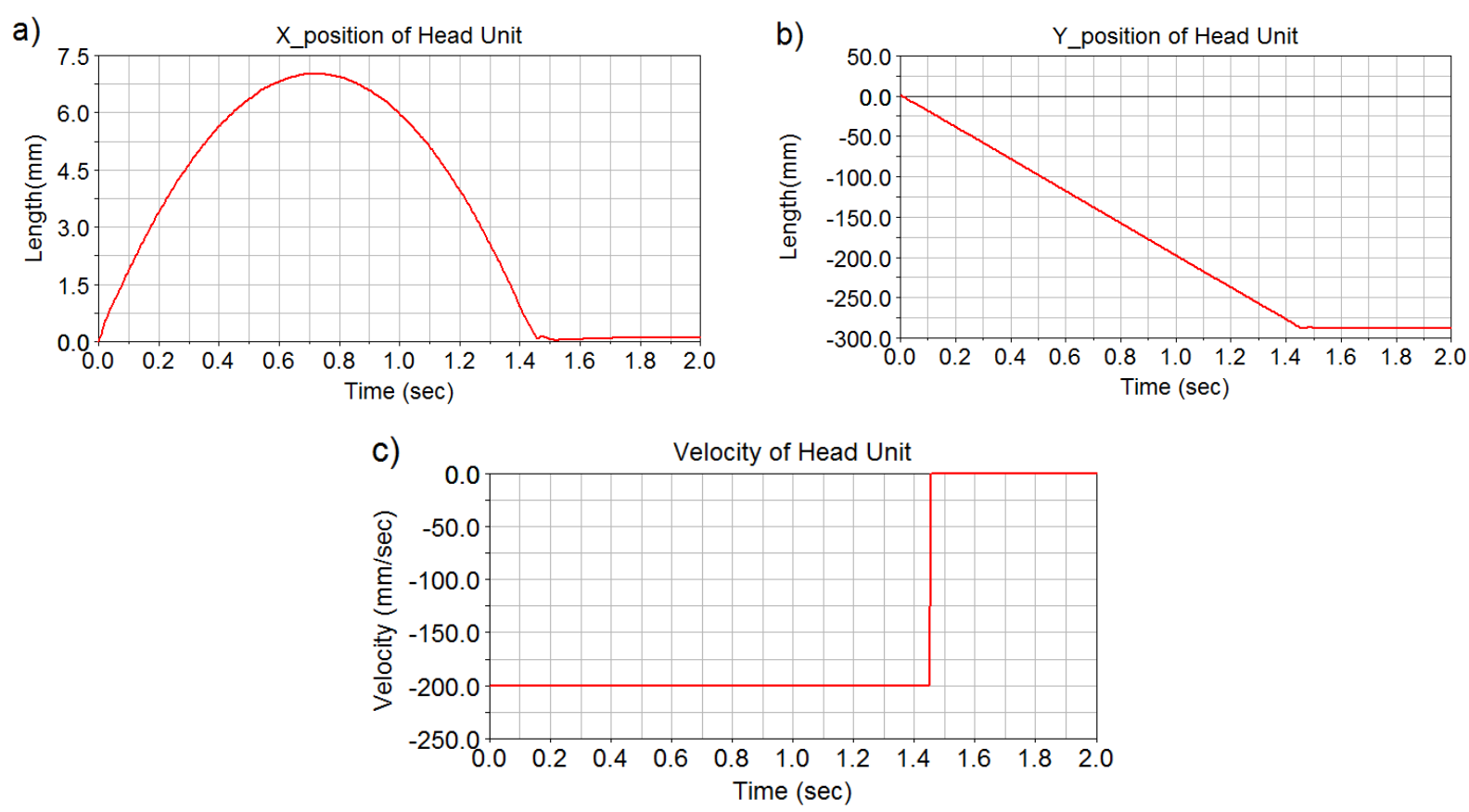

Fig. 9. The simulation results in the moving process 

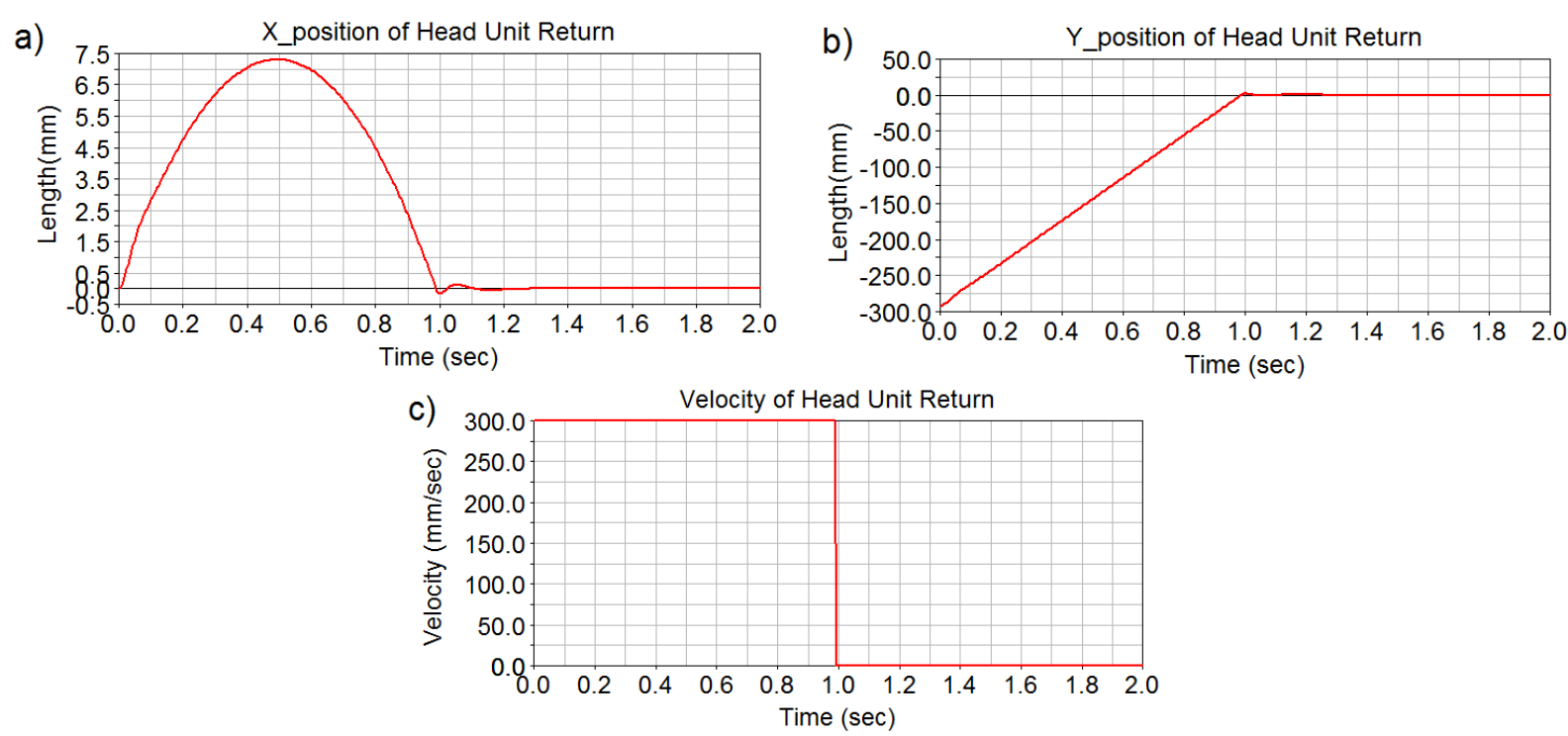

Fig. 10. The simulation results in the return process

The simulation results of the position and velocity of the head unit in ADAMS software as shown in Fig. 9 and Fig. 10 The trajectory of the head unit in X-axis and Y-axis are reflected correctly the shape of the product. Moreover, the velocity of the head unit is $200 \mathrm{~mm} / \mathrm{s}$ in the moving process and $300 \mathrm{~mm} / \mathrm{s}$ in the return process as the specifications of the machine when design. Therefore, the multi body simulation model can be used to imitate the characteristics of the flexible cutting machine.

\section{Development of a control system for the flexible cutting machine}

\subsection{The interaction between the multi-body system and the control system}

The principle of the connection between ADAMS and MATLAB/Simulink is depicted as Fig. 11. The input signal is a torque of the main servo motor and the output signals are the measured parameters of the position of the head unit. Subsequently, this model is exported to MATLAB/Simulink which have a *.m file and an adams_sys are created as shown in Fig. 12. It is also generates a command file (*.cmd) and a dataset file (*.adm), which will be used during the simulation process. The adams_sys block is created based on the information from the *.m file. With these file, the control system block will be created in MATLAB/Simulink.

\subsection{Controller design}

The PID control method can be applied easily and is widely known in many control applications because of its simplicity and effectiveness. However, this method is insensitive to parameter changes as proportional gain $K_{P}$, integral gain $K_{I}$, derivative gain $K_{D}$ and only suitable for the linear system. Luckily, there has been extensive interest in selftuning these three controller gains. For examples, the PID self-tuning methods based on the relay feedback technique were presented for a class of systems [8]. An adaptive PID control tuning was proposed to cope with the control problem for a class of uncertain chaotic systems with external disturbance [9]. Moreover, sliding mode control (SMC) is one of the popular strategies to deal with uncertain control systems [10], [11]. The main feature of SMC is the robustness against parameter variations and external disturbances. Consequently, the adaptive PID with SMC control system (ASMP) can be updated online the PID gains with an adequate adaptation mechanism that is adapted with variations of system parameters and external disturbances. In this work, the ASMP is proposed to control the rolling process of machine. Fig. 13. shows the block diagram of the adaptive SMC with PID control system for the flexible cutting machine.

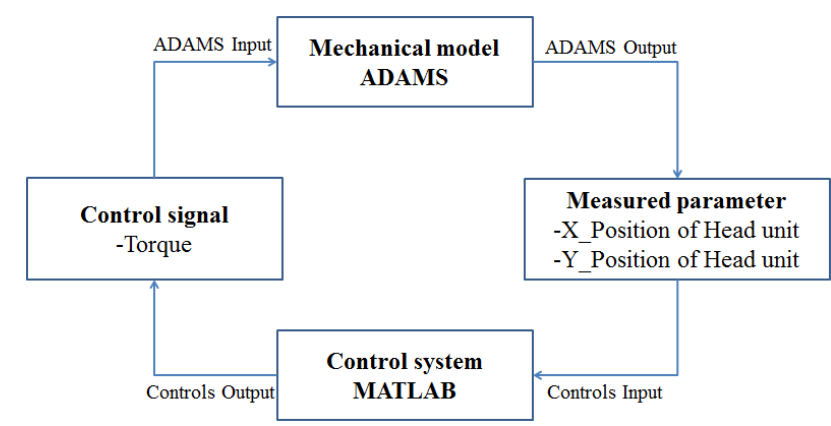

Fig.11. The principle of the connection between ADAMS and MATLAB/Simulink

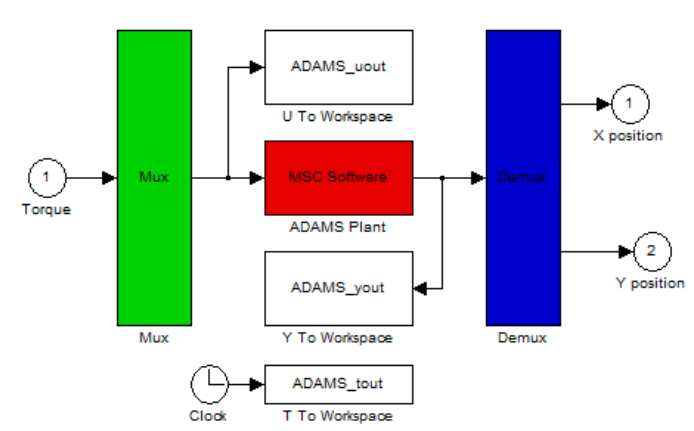

Fig.12. The adams_sub block 


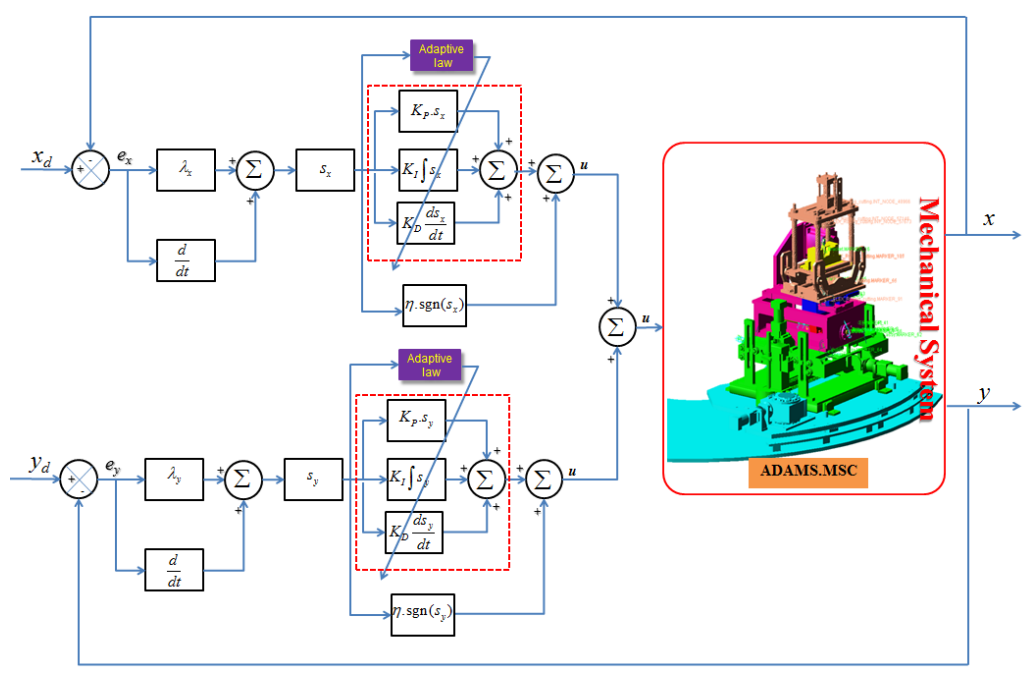

Fig. 13. Proposed ASMP control system scheme.

The flexible cutting machine is considered as a system with a single-input and multi-output. The state-space equation which expresses the head unit position can be expressed as follows:

$$
\begin{aligned}
& \dot{x}_{1}(t)=x_{2}(t) \\
& \dot{x}_{2}(t)=f_{1}(X)+b_{1}(X) u_{x}+d_{1}(t) \\
& \dot{x}_{3}(t)=x_{4}(t) \\
& \dot{x}_{4}(t)=f_{2}(X)+b_{2}(X) u_{y}+d_{2}(t)
\end{aligned}
$$

where, $X=\left(x_{1}, x_{2}, x_{3}, x_{4}\right)$ is a state variable vector that represents the position and velocity of the head unit in the $\mathrm{X}$-axis and Y-axis, respectively. The $f_{l}(X), b_{1}(X), f_{2}(X)$ and $b_{2}(X)$ are the nonlinear functions. The $d_{1}(t)$ and $d_{2}(t)$ are presented for the bounded lumped disturbances which include the system parameter variations and external disturbances. The $u_{x}$ and $u_{y}$ are the control input to control the position of the head unit in $\mathrm{X}$-axis and $\mathrm{Y}$-axis, respectively.

From (9), the mechanical model has two subsystems: the position of the head unit in $\mathrm{X}$-axis and $\mathrm{Y}$-axis. Therefore, the sliding surfaces of the two subsystems are defined as:

$$
\begin{aligned}
& s_{x}=\dot{e}_{x}+\lambda_{x} e_{x} \\
& s_{y}=\dot{e}_{y}+\lambda_{y} e_{y}
\end{aligned}
$$

In this paper, a control input $u$ were designed to control simultaneously the position of the head unit in X-axis and Yaxis. The design methods for these two controllers are similar, therefore, we will show the algorithm to drive the head unit's position in $\mathrm{X}$-axis. With the equation (10),

where $e_{x}=x_{d}-x ; x_{d}$ : desired position; $x$ : measured position; and $\lambda_{x}$ : positive constant.

Derivative (10) and substituting $\dot{x}_{2}=\ddot{x}$

$$
\dot{s}_{x}=\ddot{x}_{d}-f_{1}(X)-b_{1}(X) u_{x}-d_{1}(t)+\lambda_{x} \dot{e}_{x}
$$

The control input of PID controller is designed based on (12):

$$
u_{P I D}=\frac{1}{b_{1}(X)}\left[\ddot{x}_{d}-f_{1}(X)-d_{1}(t)+\lambda_{x} \dot{e}_{x}\right]=A B+\varepsilon
$$

where, $A=\left[K_{P} K_{I} K_{D}\right]$ is the vector of the gain of PID controller and $B=\left[s \int s \frac{d s}{d t}\right]^{T}$ is a basic vector of PID controller; $\varepsilon$ is an appropriate error. The control signal $u_{x}$ of the controller is determined as:

$$
u_{x}=u_{P I D}+u_{h}=\hat{A} B+u_{h}
$$

where, $\hat{A}=\left[\hat{K}_{P} \hat{K}_{I} \hat{K}_{D}\right]$ is estimated value of vector A; $u_{h}$ : control signal of the auxiliary controller. 
Substituting (14) into (12) yields

$$
\begin{aligned}
\dot{s}_{x} & =\ddot{x}_{d}-f_{1}(X)-b_{1}(X)\left[\hat{A} B+u_{h}\right]-d_{1}(t)+\lambda_{x} \dot{e}_{x} \\
& =b_{1} A B+b_{1} \varepsilon-b_{1} u_{h}
\end{aligned}
$$

where $A=A-\hat{A}$ is estimation error. In order to prove the stability, the Lyapuvov function can be used.

$$
V=\frac{1}{2} s_{x}^{2}+\frac{1}{2 \gamma} A^{2}
$$

Derivative (16) yields:

$$
\begin{aligned}
\stackrel{\square}{V} & =s_{x} s_{x}+\frac{1}{\gamma} A A \\
& =s_{x}\left(b_{1} A B+b_{1} \varepsilon-b_{1} u_{h}\right)+\frac{1}{\gamma} A A \\
& =\left(s_{x} b_{1} B+\frac{1}{\gamma} A\right) A+s_{x} b_{1} \varepsilon-b_{1} u_{h} s_{x} \leq 0
\end{aligned}
$$

From (17), we have

$$
\begin{array}{r}
\left(s_{x} b_{1} B+\frac{1}{\gamma} A\right)=0 \\
A=-\hat{A}=-\gamma s_{x} b_{1} B
\end{array}
$$

Hence,

The three PID gains $\left(K_{P}, K_{I}\right.$, and $\left.K_{d}\right)$ are on-line updated by the following adaptive laws:

$$
\begin{aligned}
& \hat{K}_{P}=\gamma s_{x} b_{1} s_{x}, \\
& \hat{K}_{I}=\gamma s_{x} b_{1} \int s_{x}, \\
& \hat{K}_{D}=\gamma s_{x} b_{1} \frac{d s}{d t}
\end{aligned}
$$

Considering (17)

$$
\stackrel{\square}{V}=s_{x} b_{1} \varepsilon-b_{1} u_{h} s_{x} \leq 0
$$

With the auxiliary controller : $u_{h}=\eta \operatorname{sgn}(s)$; the sign function

$$
\text { Where: } \operatorname{sgn}(s)=\left\{\begin{array}{c}
1 \text { if } s>0 \\
0 \text { if } s=0 \\
-1 \text { if } s<0
\end{array}\right.
$$

We have :

$$
\begin{aligned}
& \stackrel{\square}{V}=s_{x} b_{1} \varepsilon-b_{1} \eta \operatorname{sgn}(s) s_{x} \\
& \quad=s_{x} b_{1} \varepsilon-b_{1} \eta\left|s_{x}\right|<b_{1}\left|s_{x}\right|(|\varepsilon|-\eta)<0 \\
& \Rightarrow \eta>|\varepsilon|,
\end{aligned}
$$

Equation (17) proves that the sliding surface is stability. The control input for controlling the flexible cutting machine is composed of the position control input $u_{x}$ and $u_{y}$ as:

$$
u=u_{x}+u_{y}
$$

\section{Simulation results}

In this section, the co-simulation is implemented between the multi-body system and the ASMP controller. First of all, the proposed ASMP is applied for tracking control of the head unit of the roll forming machine, and the comparisons with PID control are presented in Fig. 14. From this figure, we can see that both PID and ASMP have the overshoot and settling time is nearly zero, and the steady state error has satisfied the stable control criteria, and disturbances are perfectly eliminated very fast responses to overcome the initial error conditions. However, the proposed ASMP control obtained much better tracking performance than PID control, indeed, the error tracking of ASMP control is smaller than PID 
control in the X-direction and Y-direction. Consequently, the ASMP controller approach is suitable for the roll forming machine which robust regardless of the system parameter and disturbance variations strategy.

Furthermore, the simulation results of the virtual prototype machine were conducted to derive the important parameters to optimize the machine prototype. Fig. 15 shows the dynamic simulation of the head unit, it was verified accurately with the specification of machine. Moreover, the value force and torque of the ball-screw and the head unit is shown in Fig. 16. The force of the ball screw impact to the head unit to implemented the rolling process is maximum to $750 \mathrm{~N}$, as illustrated in Fig. 16a. To make the head unit moving followed the curved part of the product, the largest contact force is $130 \mathrm{~N}$, as presented in Fig. 16b. Fig. 16c demonstrated the maximum torque to the ball screw is nearly $2000 \mathrm{~N}-\mathrm{mm}$. Therefore, these results were considered to support design engineers to decide the best parameters for the machine.

a)

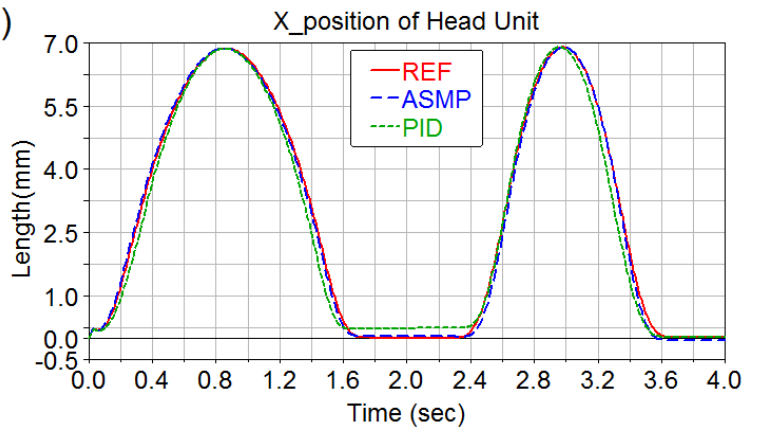

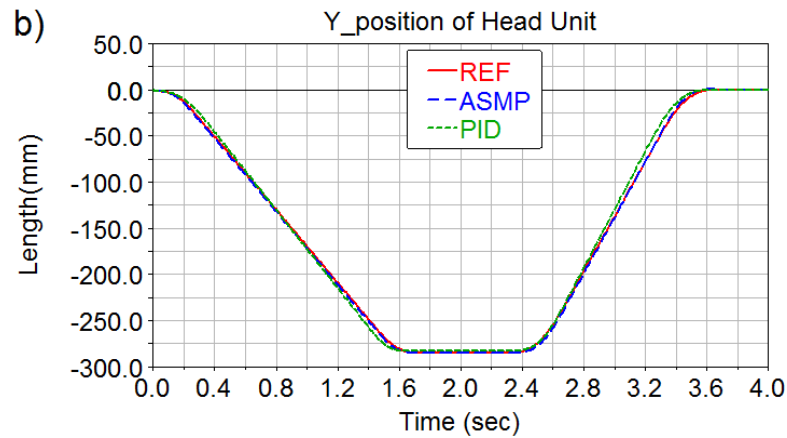

Fig. 14. Tracking the performance of the head unit of roll forming machine

a)

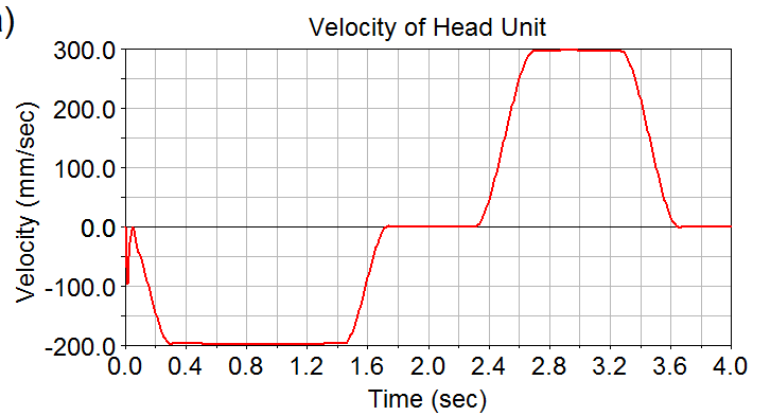

C)

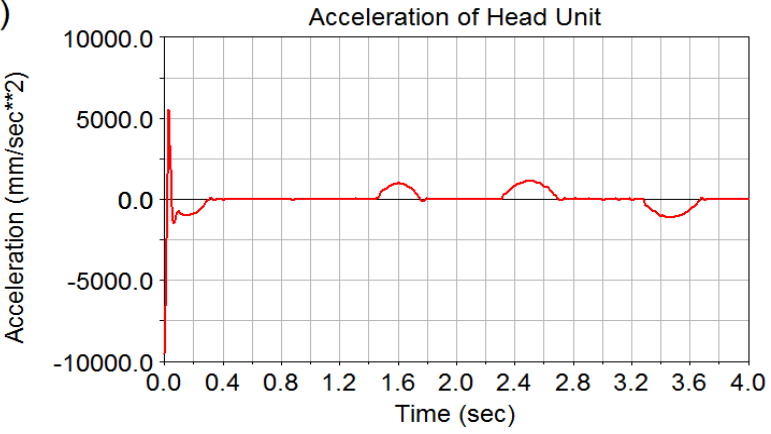

b)

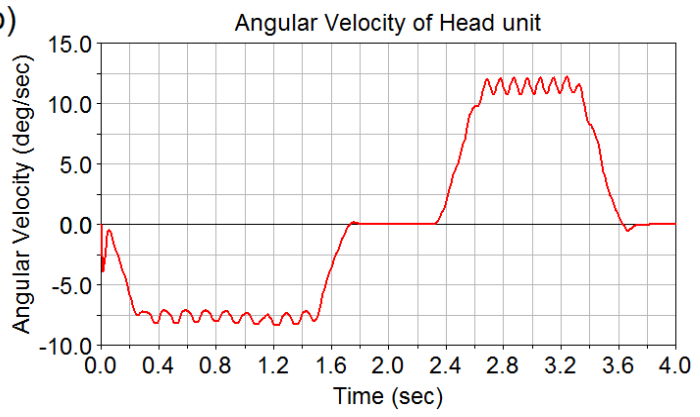

d)

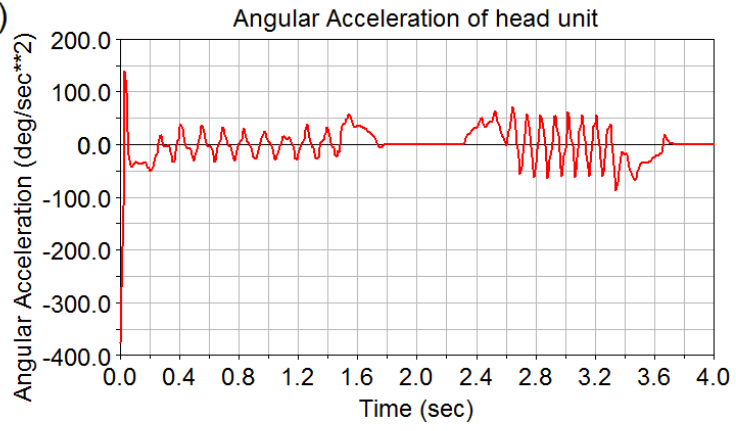

Fig. 15. The dynamic behavior of the head unit
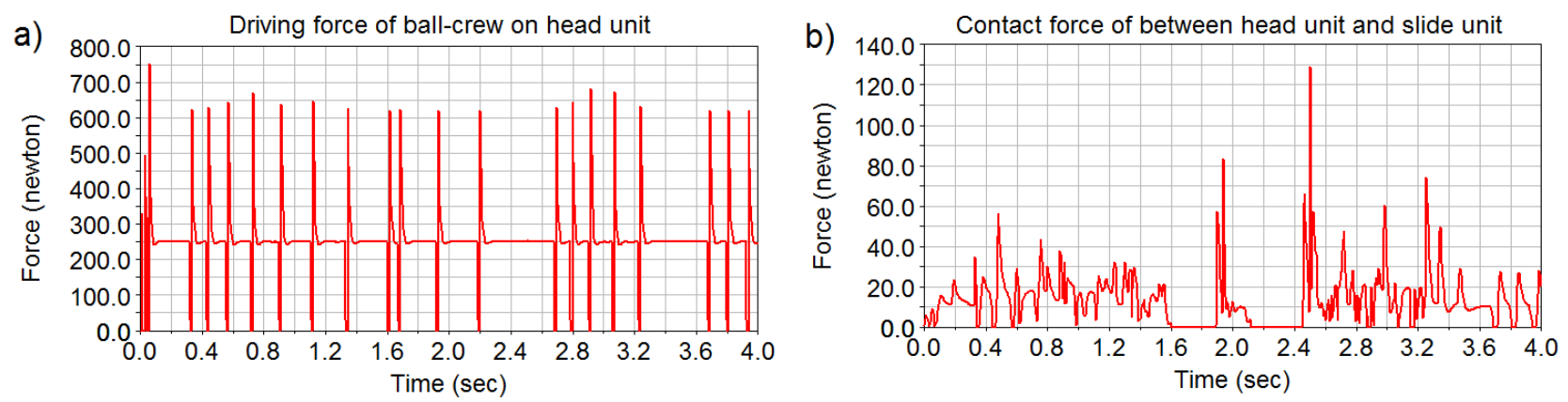


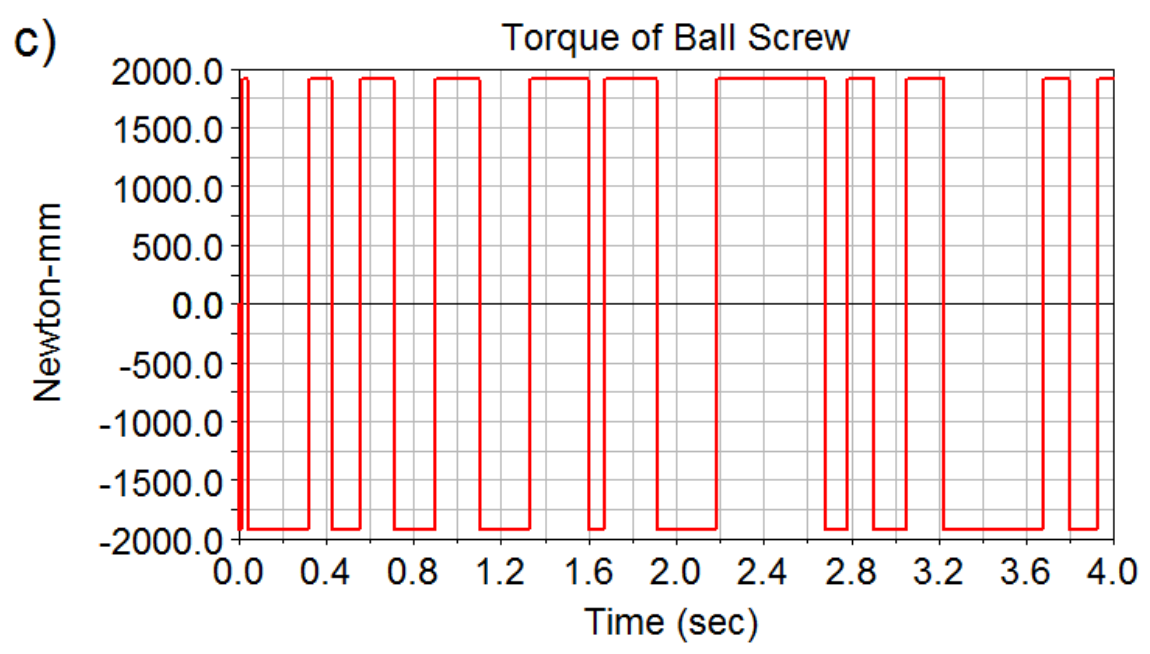

Fig. 16. The force of main components in the roll forming machine

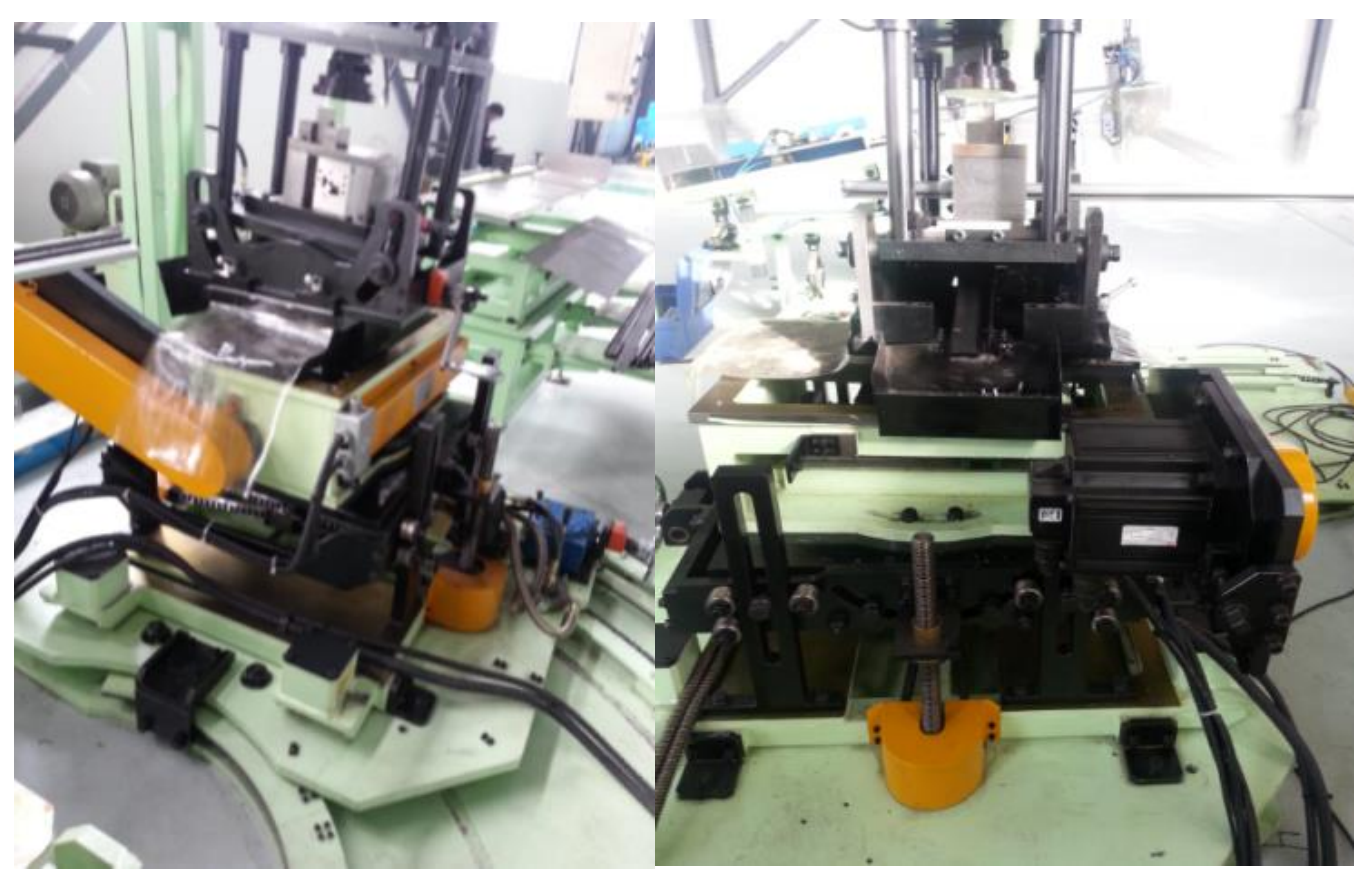

Fig. 17. Physical prototype of the flexible cutting machine for roll forming product.

\section{Conclusion}

In this paper, the flexible cutting machine for roll forming product was designed and simulated, based on a multibody system and the implementation of modern control technology. The mechanical model of the flexible cutting machine is established with the concept ideas to response the cutting requirements of roll forming product. Subsequently, the modelling of machine was transferred to ADAMS environment for analysis the dynamic behavior and the ASMP controller was designed for controlling the nonlinear process movement of the machine in MATLAB/Simulink. The virtual prototype simulation has already performed that the ASMP controller robustly against disturbance, increased productivity by reducing downtime, the virtual mechanical model could precisely described the dynamic behavior of the flexible cutting machine. Significantly, the approach can support the designers to modify the mechanical and control designs to eliminate errors and increase the accuracy. Therefore, this work is expected as a contribution to improving the prototype's performance of the flexible cutting machine.

In spite of the simulation models are useful, physical experiments to verify the simulation and optimization results are indispensable. Fig. 17 shows the physical prototype is established in the company. The verification of the proposed ASMP method will be implemented in this test and the results of the experimental study will be presented in a future paper. 


\section{References}

[1] C. Brecher, M. Weck, S. Witt Y. Altintas. (2005). Virtual Machine Tool CIRP Annals - Manufacturing Technology, vol. 54 , no. 2 , pp. $115-138$

[2] Catalin Alexandru, Claudiu Pozna. (2009), Dynamic Modeling and Control of the Windshield Wiper Mechanisms, Wseas transactions on systems, vol. 8, no. 7

[3] Zouhaier Affi, Lotfi Romdhane. (2005), ADAMS/Simulink interface for Dynamic Modeling and Control of Closed Loop Mechanisms, Proceedings of the 7th WSEAS international conference on Automatic control, modeling and simulation, USA, ISBN:960-8457-12-2, pp. 353-356

[4] Zdenek Hajicek. (2015), Simulation of virtual machine tool during the development phase, Proceedings of the 26th DAAAM International Symposium, ISSN 1726-9679, ISBN 978-3-902734-07-5, pp. 0948-0954

[5] Park Hong Seok \& Le Ngoc Tran. (2011), A 3d simulation system for mobile harbourcrane based on virtual prototyping technology, Proceedings of the 22nd International DAAAM Symposium, ISSN 1726-9679, ISBN 9783-901509-83-4, pp. 0035-0036

[6] Zoran Pandilov, Andrzej Milecki, Amadeusz Nowak, Filip Górski, Damian Grajewski Damir Ciglar, Tihomir Mulc \& Miho Klaić. (2016), Virtual Modelling and Simulation of a CNC Machine Feed Drive System, Transaction of FAMENA, vol. 39, no. 4, pp. 37-54

[7] Dan Negrut, Brett Harris. (2001), ADAMS Theory in a Nutshell

[8] A.Leva. (1993), PID autotuning algorithm based on relay feedback, IEE Porc-Control Theory Appl., vol. 140, pp. 328-337

[9] W. D. Chang \& J.J.Yan. (2005), Adaptive robust PID controller design based on a sliding mode for uncertain chaotic systems, Chaos Solitions \& Fractals, vol. 26, pp. 167-175

[10] K. D.Young, V. I. Utkin \& Ü. Özgüner. (1999), A control engineer's guide to sliding mode control, IEEE Trans. Control Sys. Tech, vol. 7, pp. 328-342

[11] A. S.I.Zinober. (1994), Variable Structure and Lyapnuov Control, Berlin: Springer-Verlag, 1994 